\title{
Fast instability caused by electron cloud in combined function magnets
}

\author{
S. A. Antipov \\ The University of Chicago, Chicago, Illinois 60637, USA \\ P. Adamson, A. Burov, S. Nagaitsev, and M.-J. Yang \\ Fermilab, Batavia, Illinois 60510, USA \\ (Received 14 December 2016; published 10 April 2017)
}

\begin{abstract}
One of the factors which may limit the intensity in the Fermilab Recycler is a fast transverse instability. It develops within a hundred turns and, in certain conditions, may lead to a beam loss. The high rate of the instability suggests that its cause is electron cloud. We studied the phenomena by observing the dynamics of stable and unstable beams, simulating numerically the buildup of the electron cloud, and developed an analytical model of an electron cloud driven instability with the electrons trapped in combined function dipoles. We found that beam motion can be stabilized by a clearing bunch, which confirms the electron cloud nature of the instability. The clearing suggest electron cloud trapping in Recycler combined function magnets. Numerical simulations show that up to $1 \%$ of the particles can be trapped by the magnetic field. Since the process of electron cloud buildup is exponential, once trapped this amount of electrons significantly increases the density of the cloud on the next revolution. In a Recycler combined function dipole this multiturn accumulation allows the electron cloud to reach final intensities orders of magnitude greater than in a pure dipole. The estimated resulting instability growth time of about 30 revolutions and the mode frequency of $0.4 \mathrm{MHz}$ are consistent with experimental observations and agree with the simulation in the PEI code. The created instability model allows investigating the beam stability for the future intensity upgrades.
\end{abstract}

DOI: 10.1103/PhysRevAccelBeams.20.044401

\section{FAST INSTABILITY}

In 2014 a fast transverse instability was observed in the proton beam of the Fermilab Recycler. The instability acts only in the horizontal plane and typically develops in 20-30 revolutions. The instability also has the unusual feature of selectively impacting the only first batch above the threshold intensity of $\sim 4 \times 10^{10}$ protons per bunch (Fig. 1). These peculiar features suggest that a possible cause of the instability is an electron cloud. Earlier studies by Eldred et al. [1] indicated the presence of an electron cloud in the recycler. The possibility of its trapping in the recycler combined function magnets was suggested Lebedev [2].

The fast instability seems to be severe only during the start-up phase after a shutdown, with significant reduction being observed after beam pipe conditioning during the operation [3]. It does not limit the current operation with slip-stacking up $700 \mathrm{~kW}$ of beam power, but may pose a challenge for a future Proton Improvement Plan II (PIP-II) intensity upgrade [4].

Published by the American Physical Society under the terms of the Creative Commons Attribution 4.0 International license. Further distribution of this work must maintain attribution to the author(s) and the published article's title, journal citation, and DOI.

\section{ELECTRON CLOUD TRAPPING}

The most likely candidates for the location of an electron cloud in the recycler are its combined function magnets. They occupy about $50 \%$ of the ring's circumference. In a combined function dipole the electrons of the cloud move along the vertical field lines, but the gradient of the field creates a condition for a "magnetic mirror" (Fig. 2) —an electron will reflect back at the point of maximum magnetic field $B_{\max }$ if the angle between the electron's velocity and the normal to the field lines is less than (for the derivation see, for example, $[5,6])$

$$
\theta_{\max }=\cos ^{-1}\left(\sqrt{B_{0} / B_{\max }}\right) .
$$

The particles with angles $\theta<\theta_{\max }$ are trapped by the magnetic field. For recycler magnets (Table I), Eq. (1) gives a capture of $\sim 10^{-2}$ particles of an electron cloud, assuming the particles start at the beam center, where the field $B=B_{0}$, and their angular distribution is uniform.

Let us look at the process of electron cloud trapping in more detail. Let us assume that a proton bunch train has created some electron cloud and consider the last two bunches of the train. The first bunch kicks the electrons of the cloud, typically supplying them with an energy of the order of one $100 \mathrm{eV}$. The electrons drift along the magnetic field in the vacuum chamber, finally reaching its walls and producing secondary electrons with the energies of a few 


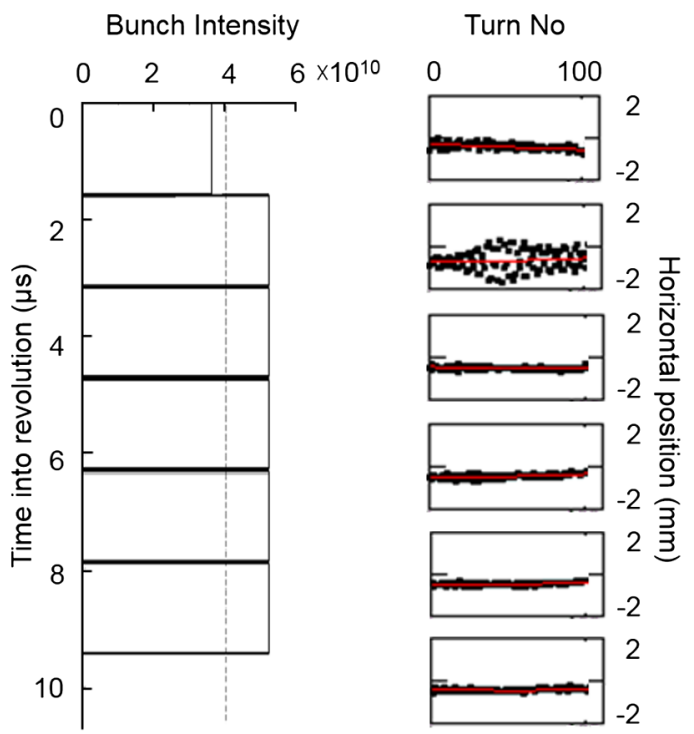

FIG. 1. The first batch above the threshold intensity suffers the blowup after injection into the ring [3]. The bunch intensities of six consecutive injections in the recycler are shown on the left. The first bunch train (top) has the intensity below the threshold (dashed line), and the following injections are above the threshold. The vertical axis shows the injection position of the bunch trains in the ring. Right: turn-by-turn measurements of beam center positions of the corresponding bunch trains during the first 100 turns after the injection. Only the first batch above the threshold intensity becomes unstable.

$\mathrm{eV}$ [7]. In the absence of the beam these secondary electrons will reach the aperture and, perhaps after a few elastic reflections, will eventually get absorbed by the vacuum chamber. But the next proton bunch can stop a fraction of the secondaries, reducing their angle $\theta$ to below the critical value (1). These electrons will remain trapped in the magnetic field after the beam is gone.

The presence of the second bunch is necessary to stop the particles, created by the first one, and therefore a single

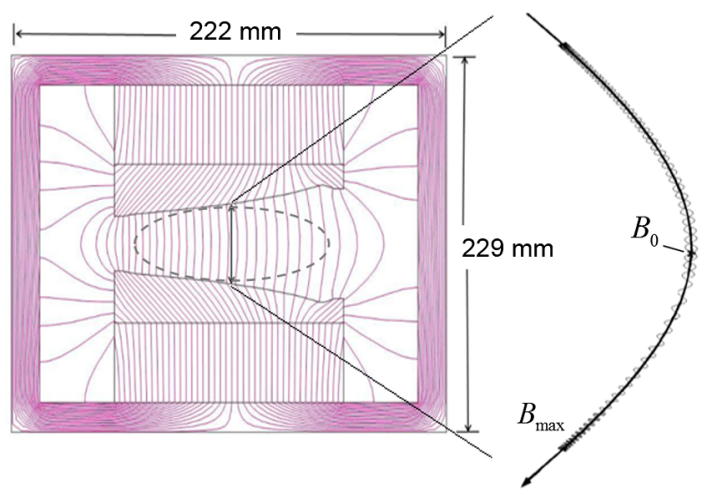

FIG. 2. Electron cloud can get trapped by a magnetic field of a combined function magnet. Image shows the field lines inside a recycler permanent combined function dipole; the gray dashed line represents the vacuum chamber.
TABLE I. Recycler parameters for simulation in PEI.

\begin{tabular}{lc}
\hline \hline Beam energy & $8 \mathrm{GeV}$ \\
Machine circumference & $3.3 \mathrm{~km}$ \\
Batch structure & 80 bunches, $5 \times 10^{10} \mathrm{p}$ \\
Tunes: $x, y, z$ & $25.45,24.40,0.003$ \\
rf harmonic number & 588 \\
rms bunch size: $x, y, z$ & $0.003,0.003,0.4 \mathrm{~m}$ \\
Maximum secondary emission yield & 2.1 at $250 \mathrm{eV}$ \\
Probability of elastic reflection & 0.5 at $0 \mathrm{eV}$ \\
Density of ionization $\mathrm{e}^{-}$ & $10^{4} \mathrm{~m}^{-1}\left(\right.$ at $\left.10^{-8} \mathrm{Torr}\right)$ \\
$B$-field and its gradient & $0.138 \mathrm{~T}, 0.34 \mathrm{~T} / \mathrm{m}$ \\
Beam pipe & elliptical, $100 \times 44 \mathrm{~mm}$ \\
\hline \hline
\end{tabular}

bunch cannot trap the cloud. Instead it clears the space, kicking the cloud to the physical aperture. The secondary electrons, created in the process, will eventually reach the vacuum chamber and be absorbed. This clearing bunch can be used to indicate the presence of the electron cloud [8] or to bring the electron cloud density below the threshold, stabilizing the beam.

The long-term confinement of the trapped electron cloud can be affected by two effects: longitudinal drift and scattering. The drift is caused by the horizontal derivative of the magnetic field $B^{\prime}=d B / d x$. The longitudinal drift velocity is

$$
v_{d}=\frac{1}{2} \omega_{c} r_{c}^{2} \frac{B^{\prime}}{B_{0}}
$$

where $\omega_{c}=e B_{0} /\left(m_{e} c\right)$ is the cyclotron frequency, $B_{0}$ is the dipole magnetic field component, and $r_{c}$ is the radius of the orbit. If the cloud drifts a distance $l_{d}$ comparable with the magnet length $L_{\text {dip }}$ it may escape the magnet and decay. For the Fermilab Recycler $v_{d}<2 \times 10^{3} \mathrm{~m} / \mathrm{s}, L_{\text {dip }}=5 \mathrm{~m}$ and the lifetime is $\sim 10^{-3} \mathrm{~s}$.

In general, the lifetime of trapped electrons may be also limited by scattering on each other and on the residual gas. For the scattering on the other electron cloud particles the Coulomb cross section $\sigma_{C}$ can be estimated as [see Eq. (41.7) in [9]]

$$
\begin{aligned}
\sigma_{C} & =16 \pi r_{e}^{2}\left(\frac{m_{e} c^{2}}{\varepsilon}\right)^{2} \Lambda_{C}, \\
\Lambda_{C} & =\ln \left(1 / \chi_{\min }\right)
\end{aligned}
$$

where $r_{e}$ is the classical electron radius, $\varepsilon$ is the electron energy, $c$ is the speed of light, $\Lambda_{C}$ is the Coulomb logarithm, and $\chi_{\min }$ is the minimal scattering angle. $\chi_{\min }$ can be estimated as $\chi_{\min } \sim \theta_{\max }$, since the scattering does not lead to a particle loss if it stays within the trapping cone $\theta<\theta_{\max }$. Then for the electron energies $\varepsilon \sim 1-10 \mathrm{eV}$ the cross section is small $\sigma_{C}<10^{-17} \mathrm{~m}^{2}$.

According to the experimental measurements [10], the scattering cross section for many residual gases is of the 
order of $10^{-15} \mathrm{~cm}^{2}$ at the energies $\varepsilon<10 \mathrm{eV}$. Combining the two scattering effects we obtain a lifetime $\sim 1 \mathrm{~ms}$ for the electron cloud density $n_{e}<10^{13} \mathrm{~m}^{-3}$ and the residual gas pressure $P \sim 10^{-8}$ Torr.

Since all the loss mechanisms result in the lifetime much larger than the revolution period of $11 \mu \mathrm{s}$, all of the trapped cloud will be present on the next turn. It will act as the new seed electrons, and can lead to a higher electron cloud density on the next revolution.

\section{NUMERICAL SIMULATION}

We simulated electron cloud buildup over multiple revolutions in a recycler dipole using the PEI code [11]. The code simulates the buildup and 2D transverse coupled motion of the electron cloud and the beam. The electron cloud is represented by an ensemble of macroparticles of a constant weight, and the beam by a series of rigid bunches with Gaussian transverse shape. The beam-cloud interaction is calculated using the Basetti-Erskine model [12]. The ring was modeled as a linear transfer matrix with one interaction point, representing a combined function magnet. The input parameters of the simulation are summarized in Table I.

The main source of primary electrons in the recycler is the collisional ionization of residual gas by the beam. To simulate it we put the seed electrons at the beam center with their linear density following $\lambda\left[\mathrm{m}^{-1}\right] \sim 6 N_{b} P[$ Torr $]$, where $N_{b}$ is the number of protons in a bunch [13].

The model of secondary emission includes true secondary and elastically reflected electrons and assumes normal incidence [14]. In a dipole field, however, an electron hits the wall of a vacuum chamber at an angle. That increases the time the electron spends near the wall surface and consequently the secondary emission yield (SEY). Experimental data on angular dependence of SEY fits an empirical formula:

$$
\mathrm{SEY}=\operatorname{SEY}_{0}\left\{1+a_{1}\left[1-\cos (\theta)^{a_{2}}\right]\right\}
$$

where $a_{1}$ and $a_{2}$ are material specific parameters and $\mathrm{SEY}_{0}$ is the yield measured at normal incidence [15].

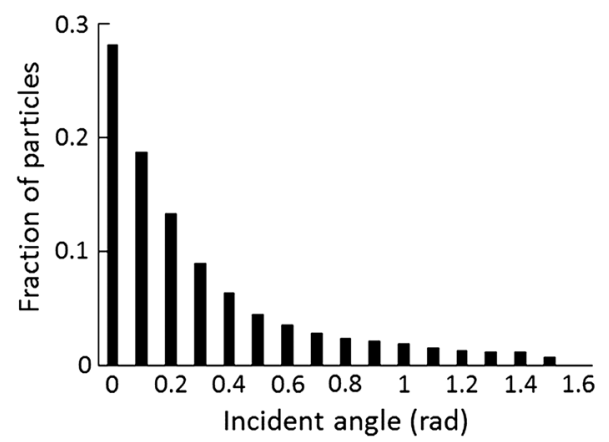

FIG. 3. Most particles hit the vacuum chamber at small angles to the normal.

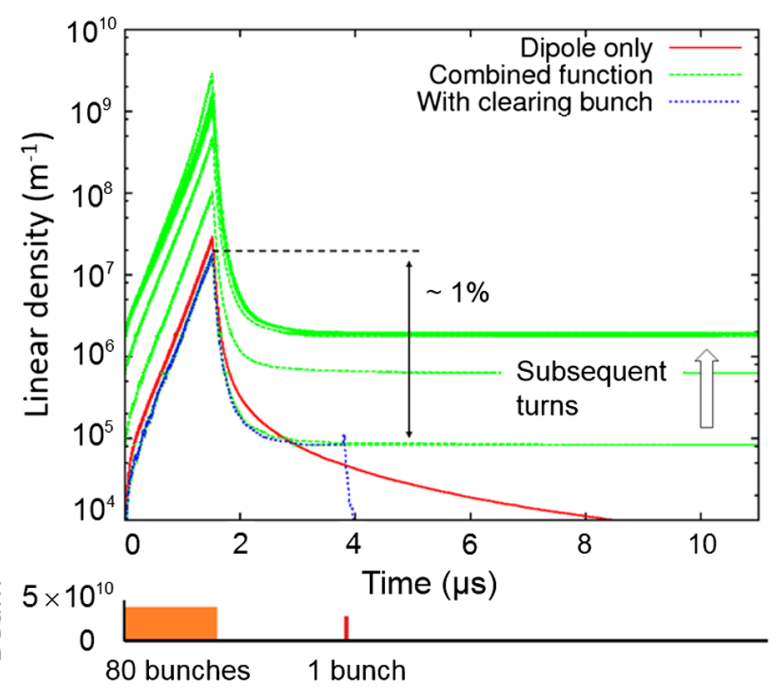

FIG. 4. In a combined function magnet the electron cloud accumulates over many revolutions, reaching much higher line density, than in a pure dipole. A clearing bunch destroys the trapped cloud, preventing the accumulation. Results of a numerical simulation using the PEI code.

For a simple estimate one can use $a_{1}=0.26, a_{2}=2$. Then for the Fermilab Recycler combined function dipoles the simulated mean incident angle is $15^{\circ}$ (Fig. 3) and the resulting increase of SEY, according to Eq. (4) is about $5 \%$.

For a pure dipole field, the cloud rapidly builds up during the passage of the bunch train and then decays back to the initial ionization electron density in about 300 rf buckets, or $\sim 6 \mu$ s (Fig. 4). When the field gradient is added, up to $1 \%$ of the electron cloud stays trapped, increasing the initial density on the next revolution. The final density, which the cloud reaches after $\sim 10$ revolutions, can be as high as 2 orders of magnitude greater than in the pure dipole case (Fig. 4). The resulting cloud distribution is a stripe along the magnetic field lines, with higher particle density being closer to the walls of the vacuum chamber (Fig. 5).

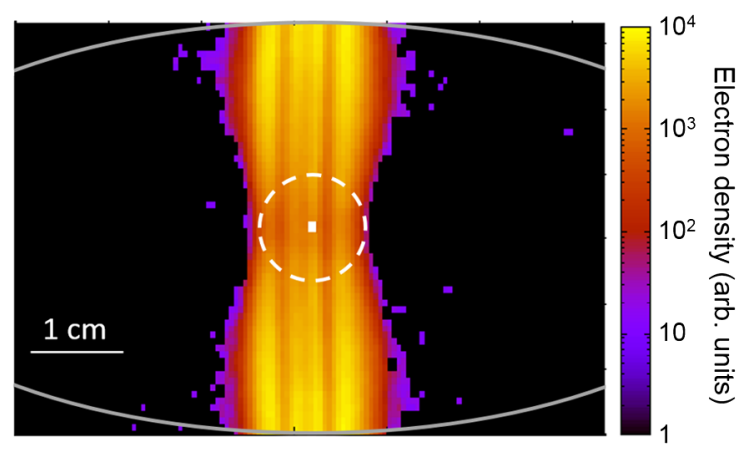

FIG. 5. Electron cloud forms a stripe inside the vacuum chamber; beam center and its 2- $\sigma$ boundary are shown in white. Results of a numerical simulation using the PEI code. 
At lower densities $\sim 10^{-2}$ of particles are trapped, which agrees with an estimate from Eq. (1); as the density of the electron cloud increases, the trapping ratio goes down to $\sim 10^{-3}$, probably due to the space charge of electron cloud.

As mentioned above, a trapped cloud can be cleared by a single bunch following the beam at a sufficient distance. In the simulation, if a bunch of $5 \times 10^{10}$ protons is added 120 rf bucket behind the main batch, it destroys the trapped cloud, preventing the multiturn buildup (Fig. 4). First, one can see a small increase in the cloud density as the clearing bunch kicks the cloud and it reaches the vacuum chamber, producing the secondary electrons. Then, the density rapidly drops as these secondaries reach the aperture.

The multiturn electron cloud accumulation due to the trapping mechanism might play an important role in a proton ring, where the density of the primary ionization electrons is relatively low. For a positron machine of a similar energy, the amount of primary electrons is much greater due to the photoemission by synchrotron radiation. Because of the large number of primary electrons, the cloud can reach a saturation density during the passage of one bunch train. Then the presence of trapping would only slightly affect the overall picture, shifting the saturation towards the head of the batch. The recent studies at Cornell Electron Storage Ring (CESR) show that, the cloud in its combined function magnets reaches a saturation density during the passage of one positron bunch train [16].

\section{WITNESS BUNCH EXPERIMENT}

We used a clearing bunch technique, similar to that used at Cornell [8], to check whether the instability is caused by a trapped electron cloud. If a trapped electron cloud is present in the machine, a single bunch of high enough charge following the main batch will kick it and clear the aperture. This clearing of the electron cloud then can be noted by observing a change in beam dynamics.

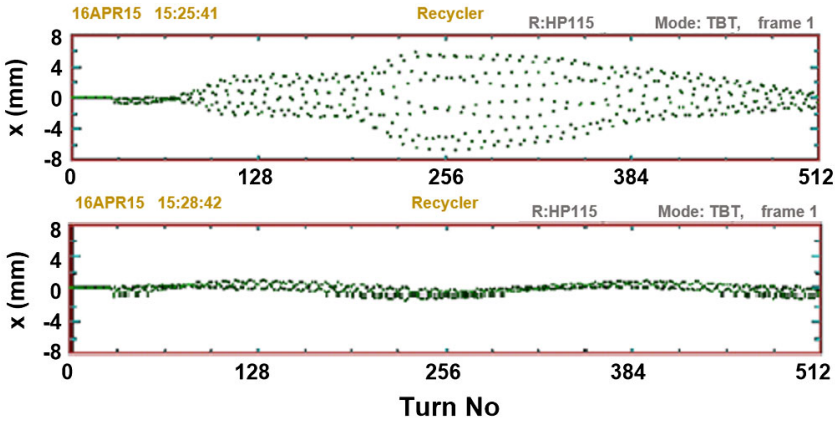

FIG. 6. Without the clearing bunch the beam of $3.6 \times 10^{12} \mathrm{p}$ blows up in about 20 turns (top); with the clearing bunch of $1 \times 10^{10} \mathrm{p}$ it remains stable (bottom). Turn-by-turn measurement of the horizontal position of the beam center.

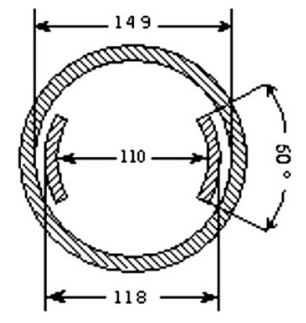

FIG. 7. Cross section of the Recycler stripline detector; dimensions in $\mathrm{mm}$ [17].

Figure 6 (top) shows the increase of beam center oscillations, measured by beam position monitors (BPMs), of an unstable batch just above the threshold intensity. The batch consists of 80 bunches of $4.6 \times 10^{10} \mathrm{p}$. The horizontal oscillations rapidly grow, leading to beam dilution and a loss of a fraction of intensity, then the beam is stabilized by the dampers. When a single clearing bunch of $\geq 1 \times 10^{10} \mathrm{p}$ is injected in the machine before the highintensity batch, the later remains stable (Fig. 6, bottom). The position of the clearing bunch does not change the picture; it can be as far as half of the ring (or $\sim 5 \mu \mathrm{s}$ ) apart from the batch, suggesting that there is a portion of the electron cloud that survives over one revolution, and it can be removed with a clearing bunch. This agrees qualitatively with the simulation of electron cloud buildup and trapping in recycler dipoles (Fig. 4).

The presence of an electron cloud provides additional focusing or defocusing, shifting the betatron tunes. Since the space charge does not change the coherent tune and the resistive wall creates a negative tune shift, a positive horizontal tune shift, if observed, would be a clear signature of the presence of the electron cloud. The tune shifts were measured with a stripline detector. The detector consists of two horizontal and two vertical $1.4 \mathrm{~m}$ long plates (quaterwave for $53 \mathrm{MHz}$ ) with $50 \Omega$ wave impedance inside a round vacuum chamber (Fig. 7). The detector has a $1 \mathrm{GHz}$ bandwidth and a linear response within $75 \%$ of the physical aperture of $110 \mathrm{~mm}$ [17].

Figure 8 depicts the betatron tune shift within the 80bunch train with respect to the first bunch, measured over 600 revolutions with a stripline detector, with the dampers off during the measurement. The positive horizontal tune shift is a clear signature of the presence of a negative charge at the beam center. The vertical tune shift is negative, indicating that the maximum density of the cloud is outside the beam, which agrees qualitatively with the simulated distribution. Figure 9 shows the simulated potential of the electron cloud, seen by the last bunch of the train (the distribution of the cloud density is depicted in Fig. 5). Its "saddle" shape near the beam center leads to horizontal focusing and vertical defocusing. When a clearing bunch is added, the tune shifts decrease, indicating a reduction of electron cloud density, which agrees with the simulation (Fig. 4). The remaining linear slope in the vertical tune shift 

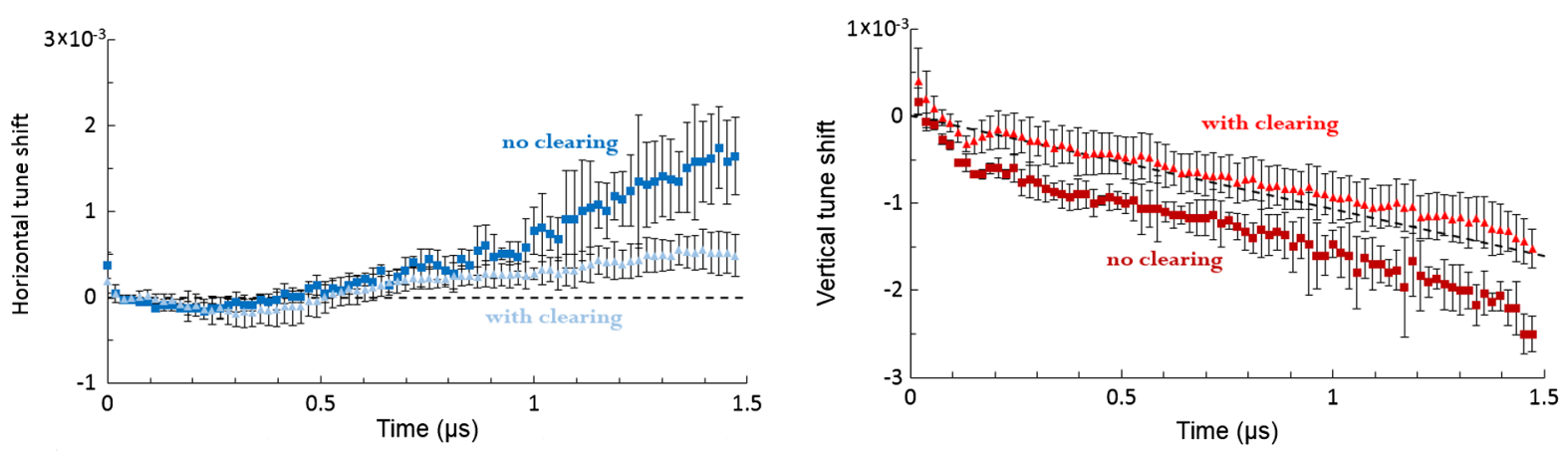

FIG. 8. Presence of the clearing bunch reduces the tune shift between the head and the tail of the high-intensity bunch train: $5 \times 10^{10} \mathrm{ppb}, 80$ bunches. The error bars represent the spread between different measurements. The dashed lines correspond to the estimated resistive wall contributions, found by extrapolating measurements at lower intensities.

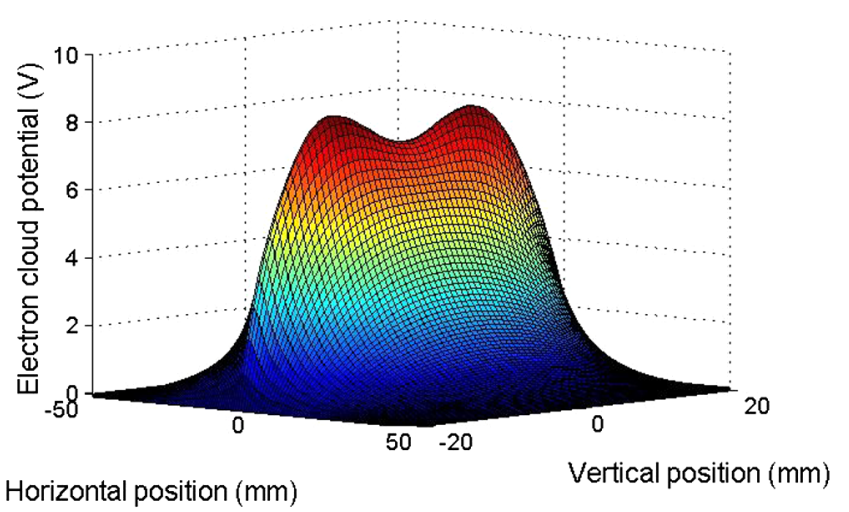

FIG. 9. Simulated potential of the electron cloud, seen by the last bunch of the train of 80 bunches, $5 \times 10^{10} \mathrm{ppb}$. The absolute value of the potential is plotted as a function of displacement from the beam center. The cloud potential decreases for small horizontal displacements and increases for small vertical ones.

is likely to be due to the resistive wall impedance. According to the recent measurements, in the recycler the vertical impedance is about 5 times larger than the horizontal [18].

\section{SIMPLE ANALYTICAL MODEL OF BEAM-CLOUD INTERACTION}

As a reactive medium, the electron cloud interacts with the beam similarly to a low- $Q$ impedance [19-21]. One of the first attempts to describe the resulting instability belongs to Burov and Dikansky in 1997 [22], who studied the cases of a field-free drift section and a bending magnet. Later Channell solved the coupled $e-p$ motion in the presence of a quadrupole magnetic field [23]. Balbekov analyzed the electron cloud instability and nonlinear effects, considering the cloud in the strong dipole field as an immobile "snake" [24]. In this chapter we study the coupled beam-cloud motion in a strong dipole field, modeling the motion of the cloud "stripe" as the mobility term, similar to the work [25]. This approach does not require making initial assumptions about the form of the electron cloud wake or its impedance.

First, consider a round coasting proton beam traveling in a ring, uniformly filled with an electron cloud. Let us denote the position of the beam centroid at an azimuthal angle $\theta$ at time $t$ as $X_{p}(t, \theta)$. Further, assume that the beam travels at a constant azimuthal velocity around the ring $\omega_{0}$ and use a smooth focusing approximation with a betatron frequency $\omega_{\beta}$.

For simplicity, one can represent the electron cloud by a cylinder of a uniform charge density $n_{e}$ and the same radius as the proton beam, located at a horizontal position $X_{e}$. Let us further assume that the total number of electrons does not change in time. Because of the vertical dipole field, the individual electrons of the cloud cannot drift horizontally, but the position $X_{e}$ can change as some regions build up and others are depleted, following the transverse motion of the proton beam. The characteristic rate of this slow motion of the electron cloud $\lambda$ is then the rate of its buildup: $\lambda \sim 1 / \tau_{\text {buildup }}$.

For small oscillation amplitudes we can assume the electron-proton interaction force to be linear in displacement. Then the coupled collective motion of the beam and the electron cloud is described by the following system of equations:

$$
\left\{\begin{array}{rl}
\left(\frac{\partial}{\partial t}+\right. & \left.\omega_{0} \frac{\partial}{\partial \theta}\right)^{2} X_{p}+\Gamma\left(\frac{\partial}{\partial t}+\omega_{0} \frac{\partial}{\partial \theta}\right) X_{p} \\
& =-\omega_{\beta}^{2} X_{p}+\omega_{p}^{2}\left(X_{e}-X_{p}\right) \\
\frac{\partial}{\partial t} X_{e} & =\lambda\left(X_{p}-X_{e}\right)
\end{array},\right.
$$

where $\Gamma$ is the rate of Landau damping. The coupling frequency $\omega_{p}$ is approximated as

$$
\omega_{p}^{2}=\frac{2 \pi n_{e} r_{p} c^{2}}{\gamma}
$$


where $r_{p}$ is the classical proton radius and $\gamma$ is the relativistic factor.

The linear damping term $\Gamma$ in Eq. (5) arises from the spread in betatron frequencies for particles oscillating with different amplitudes. The characteristic rate of the Landau damping can be estimated as

$$
\Gamma \sim \omega_{\beta} \frac{\Delta Q_{x}}{Q_{x}},
$$

where $Q_{x}$ is the horizontal tune and $\Delta Q_{x}$ is its rms spread.

Looking for solutions of Eq. (5) in a form $X_{e, p} \propto e^{-i \omega t+i n \theta}$ one obtains an equation for the mode frequency $\omega$ :

$$
-\left(\omega-n \omega_{0}\right)^{2}-i \Gamma\left(\omega-n \omega_{0}\right)+\omega_{\beta}^{2}+\frac{\omega \omega_{p}^{2}}{\omega+i \lambda}=0
$$

It can be solved perturbatively, under the assumption that

$$
\omega_{\beta}, \lambda \gg \omega_{0}, \omega_{p}, \Gamma .
$$

Solving Eq. (8) for each integer wave number $n$ one gets

$$
\omega=n \omega_{0}+\omega_{\beta}+\Delta \omega,
$$

where the small complex tune shift $|\Delta \omega| \ll \omega, \lambda, \omega_{\beta}$ is

$$
\Delta \omega \approx \frac{1}{2}\left[-i \Gamma+\frac{\omega_{p}^{2}}{\omega_{\beta}} \frac{\omega(\omega-i \lambda)}{\lambda^{2}+\omega^{2}}\right] .
$$

The imaginary tune shift in Eq. (11) consists of two parts with the first being the Landau damping term. The most unstable mode, for which $\operatorname{Im}(\Delta \omega)$ is the greatest, is $\omega_{\max }=\lambda$ and its wave number $n_{\max }$ is

$$
n_{\max }=\frac{\lambda-\omega_{\beta}}{\omega_{0}}=\frac{\lambda}{\omega_{0}}-Q_{x},
$$

and the growth rate of this mode is

$$
\gamma_{\max }=\frac{1}{2}\left(\frac{\omega_{p}^{2}}{2 \omega_{\beta}}-\Gamma\right) .
$$

The threshold electron cloud density $n_{e, \text { thr }}$ can be found from the condition $\gamma_{\max }=0$, which yields

$$
n_{e, \mathrm{thr}}=\frac{\gamma \Gamma \omega_{\beta}}{\pi c^{2} r_{p}} .
$$

Since, in general, the strength of Landau damping $\Gamma$ depends on the density of the electron cloud, this equation might have one, many, or no solutions at all [21]. In this simple model we do not consider the electron cloud's contribution to Landau damping, which may arise from the nolinear spread of the betatron tunes, created by the cloud.

In an experiment an observer will see the most unstable mode as it masks the others thanks to its higher exponential growth rate. Thus, a turn-by-turn measurement of beam position at $\theta=\theta_{0}$ will detect a tune shift of

$$
\Delta Q_{\max } \approx \frac{1}{4 Q_{x}} \frac{\omega_{p}^{2}}{\omega_{0}^{2}}
$$

Knowing the complex frequency shift $\Delta \omega$ we can find the impedance of the cloud as [see for example [26] Eq. (6.262)]

$$
Z(\omega)=Z_{0} \frac{\gamma T_{0}^{2} \omega_{\beta}}{2 \pi N r_{p}} i\left[\Delta \omega+i \Gamma / 2-\omega_{p}^{2} /\left(2 \omega_{\beta}\right)\right],
$$

where $N$ is the number of protons in the ring and $Z_{0}$ is the vacuum impedance. Because the electron cloud shifts both the coherent and the incoherent frequencies, we subtracted here the incoherent tune shift.

Knowing the impedance one can compute the wake functions using the formula (2.72) from [26]:

$$
W(z)=\frac{-2 i}{Z_{0} c} \int_{-\infty}^{+\infty} Z(\omega) e^{i \frac{\omega \bar{z}}{c}} d \omega .
$$

In the case of a bunched beam, in a rigid bunch approximation, one needs to compute $W(z)$ only in a discreet set of bunch positions $z_{k}=k c \tau_{\mathrm{rf}}$, where $\tau_{\mathrm{rf}}$ is the $\mathrm{rf}$ period.

Finally, from the impedance of the most unstable mode one can estimate the instability growth rate of a bunched beam as [27]

$$
\gamma_{b, \max } \approx-\frac{L}{C} \frac{8 \pi r_{p} N_{b} \beta_{x}}{\gamma \tau_{r f} c} \frac{\operatorname{Re}\left[Z\left(\omega_{\max }\right)\right]}{Z_{0}}-\frac{1}{2} \Gamma,
$$

where $C$ is the ring circumference and $L$ is the total length of the magnets. For the recycler $L / C \approx 1 / 2$.

\section{INSTABILITY IN THE RECYCLER}

In order to use the model and estimate the parameters of the fast instability in the recycler one needs to know the density of the electron cloud and the rate of its buildup. We obtain these quantitative parameters by measuring the betatron frequency shift and comparing it with the buildup simulations.

We injected one batch of 80 proton bunches of $5 \times 10^{10} \mathrm{ppb}$ and measured the shift of the horizontal tune 


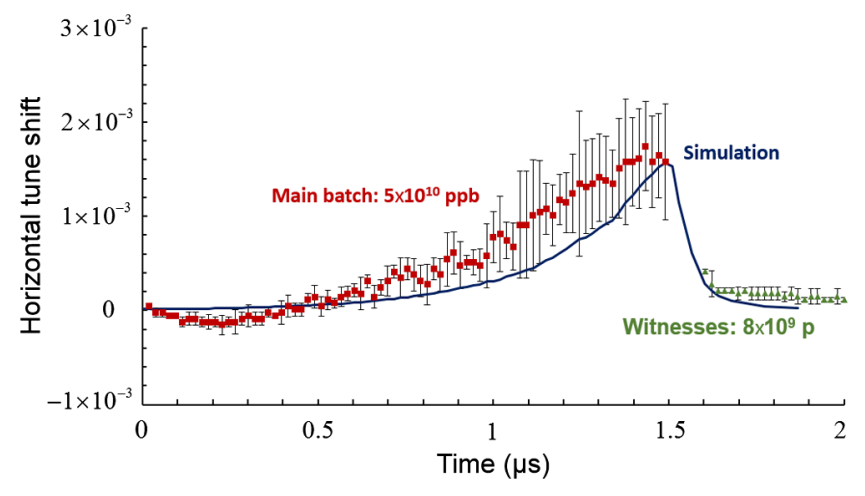

FIG. 10. Results the of electron cloud simulation agree with the measured horizontal tune shift. Beam: $5 \times 10^{10} \mathrm{ppb}, 80$ bunches, followed by one witness bunch of $0.8 \times 10^{10} \mathrm{p}$ at various positions. The gap between the high-intensity batch and the witness is due to the rise time of the injection kickers.

as a function of bunch number. Because the positive horizontal tune shift is a distinctive feature of the electron cloud, it allowed us an estimation of the cloud density. In order to check with the simulation the cloud density both within the high-intensity batch and after its passage, we put a witness bunch of low intensity $-8 \times 10^{9} \mathrm{p}$, insufficient to clear the electron cloud, at different positions behind the main batch.

The experimental results are in good agreement with the simulation (Fig. 10) and the small discrepancies may come from the multiple assumptions used in Eq. (15). The resulting dependence allows the estimation of the maximum density of the electron cloud $n_{e} \sim 6 \times 10^{11} \mathrm{~m}^{-3}$. The density increases by an order of magnitude in 40 bunches ( $800 \mathrm{~ns})$ and falls after the beam has passed in 10 bunches (200 ns). The characteristic rate of the exponential buildup is $\lambda \sim 2.65 \times 10^{6} \mathrm{~s}^{-1}$. The parameters of the model are summarized in Table II.

The most unstable mode has the frequency of about $0.4 \mathrm{MHz}$, its impedance, calculated using Eq. (16), is $20 \mathrm{M} \Omega / \mathrm{m}$ (Fig. 11). Figure 12 depicts the corresponding wake function $W(n)$ as a function of bunch number $n$. $W(n)$ fits an exponential decay curve

TABLE II. Parameters of the model.

\begin{tabular}{lcc}
\hline \hline Parameter & Symbol & Value \\
\hline Relativistic factor & $\gamma$ & 10 \\
Cyclotron frequency & $\omega_{0}$ & $0.57 \times 10^{6} \mathrm{~s}^{-1}$ \\
Betatron frequency & $\omega_{\beta}$ & $14.54 \times 10^{6} \mathrm{~s}^{-1}$ \\
Protons per bunch & $N_{b}$ & $5 \times 10^{10}$ \\
Electron cloud density & $n_{e}$ & $6 \times 10^{11} \mathrm{~m}^{-3}$ \\
$e-p$ coupling frequency & $\omega_{p}$ & $0.23 \times 10^{6} \mathrm{~s}^{-1}$ \\
Buildup rate & $\lambda$ & $2.65 \times 10^{6} \mathrm{~s}^{-1}$ \\
Chromatic tune spread & $\Delta Q_{x}$ & $2.7 \times 10^{-3}$ \\
\hline \hline
\end{tabular}

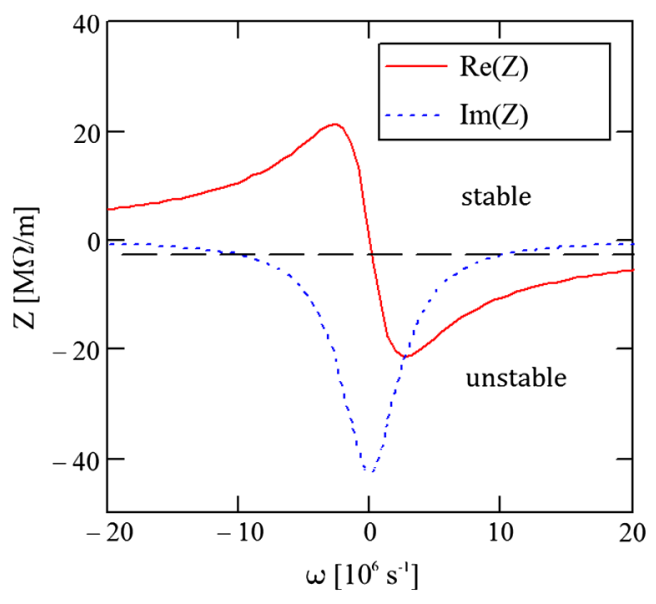

FIG. 11. Real and imaginary parts of impedance as a function of a mode angular frequency $\omega$. The dashed line represents the Landau damping. The modes with $\operatorname{Re}[Z(\omega)]$ below the dashed line are unstable.

$$
W=W_{0} \exp (-\Delta z / c \lambda), \quad \Delta z>0
$$

The estimate of the mode frequency qualitatively agrees with the simulation in the PEI code and the stripline measurement. PEI simulated the ring, completely filled with 588 bunches of $5 \times 10^{10} \mathrm{p}$. The resulting frequency is about $0.7 \mathrm{MHz}$ (Fig. 13). In the stripline measurement one batch of 80 bunches of the same charge was injected. The measured frequency was about 0.9 MHz. Both simulated and measured frequencies agree on the order of magnitude with each other and the estimate.

Using the calculated value of the real part of the impedance we can now estimate the growth rate using Eq. (13). We obtain the growth rate of $\gamma_{b, \max }=0.033$ and the characteristic time of the instability $\tau_{\max }=$ $1 / \gamma_{b, \text { max }} \approx 30$ turns.

The threshold electron cloud density is calculated using Eq. (14), $n_{e, \text { thr }}=8.2 \times 10^{10} \mathrm{~m}^{-3}$. This density is achieved at the proton intensity of about $4.5 \times 10^{10} \mathrm{ppb}$, which is also consistent with experimental observations.

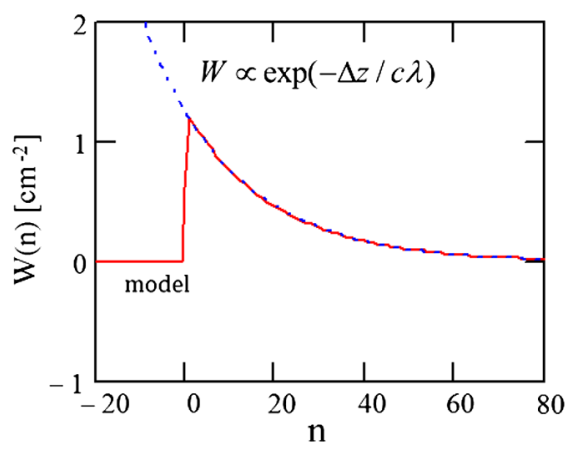

FIG. 12. Electron cloud wake falls exponentially with distance. 

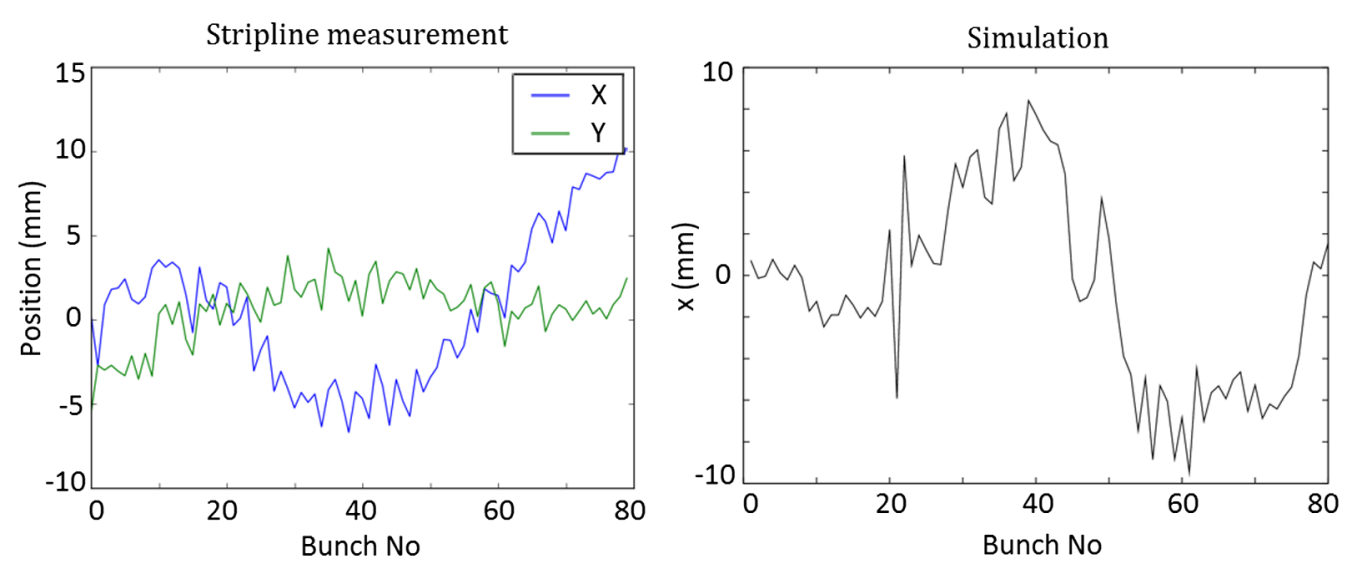

FIG. 13. Simulation in PEI and stripline measurements show an instability in the horizontal plane with a period of slightly less than the length of a batch and a frequency $<1 \mathrm{MHz}$.

\section{CONCLUSION}

A fast transverse instability in the Fermilab Recycler might create a challenge for PIP-II intensities. The PIP-II upgrade goal is nearly doubling the beam intensity and reaching $\sim 8 \times 10^{10}$ protons per bunch [4]. Since the fast instability has been already observed at the intensities as low as $4-5 \times 10^{10} \mathrm{ppb}$, understanding its nature is important for making predictions about the machine performance at higher intensities.

We have observed that the fast instability can be mitigated by injection of a single low intensity clearing bunch. This finding suggests that the instability is caused by an electron cloud and the cloud is trapped in the recycler magnets. Bunch-by-bunch measurements of the betatron tune have shown its shift towards the end of the bunch train. The tune shift decreases after the addition of the clearing bunch, which is also consistent with the electron cloud picture.

There is practically no doubt that the source of trapping is the combined function magnets, occupying around $50 \%$ of Recycler circumference. Combined function magnets are widely used in contemporary accelerators and are a technology of choice for some future machines. According to numerical simulations in PEI, $\sim 10^{-2}-10^{-3}$ of particles are trapped by the magnetic field of those magnets. That allows the electron cloud to gradually build up over multiple turns, reaching final intensities orders of magnitude greater than in a pure dipole. The results of an electron cloud buildup simulation in the recycler combined function dipoles agree qualitatively with the observed stabilization of the beam by a clearing bunch and quantitatively with the measurements of betatron tune shift. According to the simulations, the estimated cloud density is $6 \times 10^{11} \mathrm{~m}^{-3}$ on the beam axis and the characteristic times of its buildup and decay are 40 and $10 \mathrm{rf}$ periods, respectively.

We have created a simple analytical model of the transverse multibunch instability, driven by the electron cloud trapped inside the combined function magnets.
The model allows an estimation of the instability threshold, the frequency of the most unstable mode, and its growth rate. For the current parameters of the recycler beam we find the mode with a frequency of $0.4 \mathrm{MHz}$ and a growth time of 30 revolutions, which is consistent with the observations of the fast instability and the simulations in PEI. The model allows the prediction of the rate of the instability for higher intensities of the proton beam, given an estimate of the electron cloud density, which can be obtained from numerical simulations.

\section{ACKNOWLEDGMENTS}

The authors are grateful to K. Ohmi (KEK) for his help with the PEI code and to Yuri Alexakhin (FNAL) for multiple useful suggestions. Fermilab is operated by Fermi Research Alliance, LLC under Contract No. DE-AC0207CH11359 with the United States Department of Energy.

[1] J. Eldred et al., Fast transverse instability and electron cloud measurements in fermilab recycler, in Proceedings of HB2014, East-Lansing, MI, USA (JACoW, Geneva, 2014), pp. 419-427, http://accelconf.web.cern.ch/AccelConf/ HB2014/papers/proceed.pdf.

[2] V. Lebedev (private communication).

[3] J. Eldred, The High-Power Recycler: Slip-Stacking \& Electron Cloud (Fermilab, Batavia, IL, 2015).

[4] S. Holmes et al., Long term plans to increase fermilab's proton intensity to meet the needs of the long baseline neutrino program, in Proceedings of the 7th International Particle Accelerator Conference IPAC (JACoW, Geneva, 2016), pp. 3982-3985, http://accelconf.web.cern.ch/ AccelConf/ipac2016/.

[5] G. I. Budker, Plasma Physics and the Problem of Controlled Thermonuclear Reactions (Pergamon Press, New York, 1959), Vol. 3.

[6] R. F. Post, Summary of UCRL pyrotron (mirror machine) program, in Proceedings of the Second U.N. International Conference on Peaceful Uses of Atomic Energy, Paper 
A/Conf. 15/P/377, Geneva (JACoW, Geneva, 1958), Vol. 32, pp. 245-265.

[7] G. Iadarola, Ph.D. thesis [CERN Report No. CERNTHESIS-2014-04, 2014].

[8] M. G. Billing, J. Conway, E. E. Cowan, J. A. Crittenden, W. Hartung, J. Lanzoni, Y. Li, C. S. Shill, J. P. Sikora, and K. G. Sonnad, Measurement of electron trapping in the Cornell Electron Storage Ring, Phys. Rev. ST Accel. Beams 18, 041001 (2015).

[9] L. D. Landau and E. M. Lifshiz, Physical Kinetics (Fizmatlit, Moscow, 2002).

[10] A. D. MacDonald, Microwave Breakdown in Gases (Wiley, New York, 1966).

[11] K. Ohmi, CERN Report No. CERN-2013-002, 2013, pp. 219-224.

[12] M. Bassetti and G. A. Erskine, CERN Report No. CERNISR-TH-80-06, 1980.

[13] F. Zimmermann, in Handbook on Accelerator Physics, edited by A. Chao and M. Tigner (World Scientific, Singapore, 1999).

[14] E. Benedetto et al., Review and comparison of simulation codes modeling electron-cloud build up and instabilities, in Proceedings of EPAC 2004, Lucerne, Switzerland, 2004 (JACoW, Geneva, 2004), pp. 2502-2504, http://accelconf .web.cern.ch/AccelConf/e04/default.htm.

[15] M. A. Furman and M. T. F. Pivi, Probabilistic model for the simulation of secondary electron emission, Phys. Rev. ST Accel. Beams 5, 124404 (2002).

[16] J. A. Crittenden, Y. Li, S. Poprocki, and J. E. San Souice, Electron cloud simulations for the low emittance upgrade at the Cornell Electron Storage Ring, in Proceedings of NAPAC 2016, Chicago (JACoW, Geneva, 2016).
[17] J. Crisp, K. Gubrienko, and V. Seleznev, Stripline detectors for main injector, in Proceedings of National 16th Conference on Accelerators, Protvino, Russia (JACoW, Geneva, 1998).

[18] R. Ainsworth, P. Adamson, A. Burov, I. Kourbanis, and M.-J. Yang, Estimating the transverse impedance in the fermilab recycler, in Proceedings of IPAC 2016, Busan, Korea (JACoW, Geneva, 2016), pp. 867-869.

[19] K. Ohmi and F. Zimmermann, Head-Tail Instability Caused by Electron Clouds in Positron Storage Rings, Phys. Rev. Lett. 85, 3821 (2000).

[20] K. Ohmi, F. Zimmermann, and E. Perevedentsev, Wakefield and fast head-tail instability caused by an electron cloud, Phys. Rev. E 65, 016502 (2001).

[21] A. Burov, Fermilab Report No. FERMILAB-PUB-13-005AD, 2013.

[22] A. Burov and N. Dikansky, Electron cloud instabilities, in Proceedings of the International Workshop of Multibunch Instabilities Future Electron Positron Accel., Tsukuba, Japan (JACoW, Geneva, 1997).

[23] P. J. Channel, Two-stream instability model with electrons trapped in quadrupoles, J. Instrum. 4, P08008 (2009).

[24] V. Balbekov, Fermilab Report No. FERMILAB-FN-1001APC, 2015.

[25] G. Arduini et al., The electron cloud instability of the LHC beam in the CERN SPS, in Proceedings of $P A C$ 2003, Portland, OR (JACoW, Geneva, 2003), pp. 3038-3040.

[26] A. Chao, Physics of Collective Beam Instabilities in High Energy Accelerators (Wiley, New York, 1993).

[27] Yu. Alexahin, FNAL Beams-doc-4863-v1, 2015. 